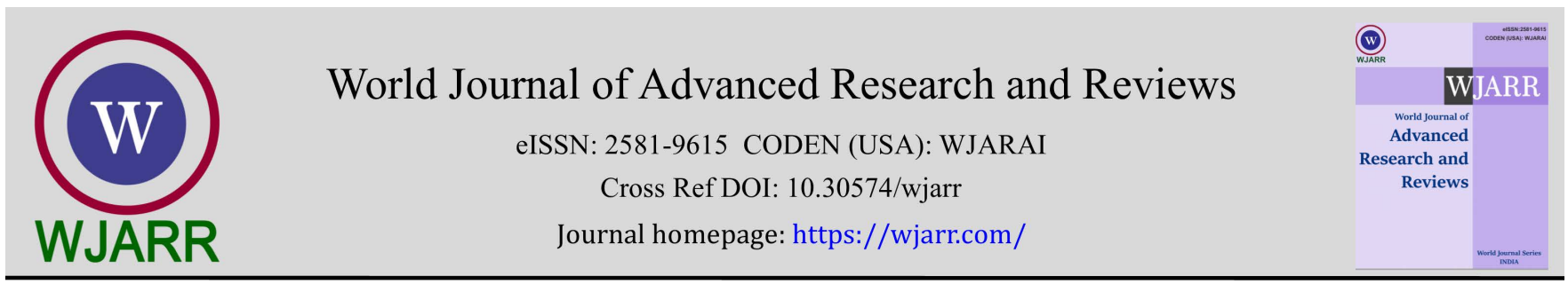

(REVIEW ARTICLE)

\title{
Iodinated contrast media
}

Sami S. Alshowiman 1, ${ }^{*}$, Abdullah H Sahrah 2, Ayman K. Alswailem 1, Saud F. Alotaibi ${ }^{1}$, Abdulaziz A. ALtowaijiri ${ }^{2}$ and Wail A. Alghathami ${ }^{1}$

${ }^{1}$ Prince Sultan Cardiac Center, Saudi Arabia.

2 Medical Services of Defense, Saudi Arabia.

World Journal of Advanced Research and Reviews, 2021, 09(01), 156-167

Publication history: Received on 20 December 2020; revised on 03 January 2021; accepted on 05 January 2021

Article DOI: https://doi.org/10.30574/wjarr.2021.9.1.0508

\begin{abstract}
Contrast media are the materials utilized in radiology to improve the visualization of certain body structures subjected to analysis in a medical image. Contrast media are, then, diagnostic drugs because they increase the visibility of organs and tissues and allow the identification of details that otherwise could not be observed. With the introduction of multidetector computed tomography the number of patients undergoing contrast studies has grown exponentially in recent years. In computed tomography the formation of the radiographic image is obtained with the use of contrast media containing iodine atoms, exploiting the different attenuation that the X-ray beam presents in crossing the anatomical districts. The Iodinated Contrast Media (ICM) are a category of numerous substances formed by even complex molecules that vary considerably in their properties, uses and toxic effects. Even though iodinated contrast agents have a good safety profile, it is necessary to understand that some patients can have severe, life-threatening allergic reactions because of the side effects of the chemicals. This paper describes the clinical pharmacology, use and adverse reactions of intravenous iodinated contrast media used in computerized tomography, offering all doctors, even non-radiologists, the opportunity for rapid updating.
\end{abstract}

Keywords: Iodinated Contrast Media, Contrast Reactions, Contrast Media, Adverse Effects, Computed Tomography (CT).

\section{Introduction}

Contrast media, also called contrast agents or contrast materials, are the substances used to enhance images of the body from the inside as they are produced from x-rays, Computed Tomography (CT), Magnetic Resonance Imaging (MRI) and Ultrasound (US) (Rusandu et al., 2020; Lim et al., 2020).

According to Brockow (2020), contrast media allow a radiologist to distinguish between normal and abnormalities from images, and the contrast materials are not the dyes that permanently change the color of internal organs, but rather the substances that temporarily change the way X-rays or other imaging tools interact with the body.

Yamaguchi et al (2017) emphasized that when inserted into the body before the imaging examination, the contrast materials make certain structures or tissues in the body look different on the images than they would appear in the absence of a contrast material. This may help the contrast materials to distinguish or "contrast" specific areas of the body from the surrounding tissues. By improving the vision of specific organs, blood vessels, or tissues, these materials help doctors diagnose medical conditions.

\footnotetext{
${ }^{*}$ Corresponding author: Sami S. Alshowiman

Prince Sultan Cardiac Center, Saudi Arabia. 
Iodine and barium sulfate compounds are used in x-rays and CT scans in imaging assays, and contrast materials can have a chemical structure that includes iodine, a naturally occurring chemical element. These contrast materials may be injected into the veins or arteries within the discs in the spine or the fluid spaces of the spine and into other body cavities (Imai et al., 2018).

Iodinated X-ray contrast media are the most commonly used drugs in diagnostic and interventional procedures. Procedures that employ contrast media have shown rapid growth. In the last two decades, the use of (CT) increased by $800 \%$ and the use of cardiac catheterization increased by 390\% (Barr et al., 2016). With the increasing use of these procedures, the number of patients receiving iodinated contrast media has also continued to increase. Given such widespread use, it is important to be aware of Associated Adverse Events (AEs) and have a good knowledge about the management of various contrast media.

Most AEs of iodinated contrast media, such as nausea, vomiting, urticaria and itching, are mild. However, severe AEs can occur, including hypotensive shock, respiratory arrest, cardiac arrest and convulsions. The incidence of these AEs has decreased considerably with the change of usage from High-Osmolar Contrast Media (HOCM) to Low-Osmolar Contrast Media (LOCM); the incidence of AEs has been reported as $5 \%$ to $15 \%$ for HOCM and $0.2 \%$ to $0.7 \%$ for LOCM (Kurihara et al., 2015; Jascintha et al., 2016). Although the overall incidence of AEs has decreased, severe AEs still continue to occur.

Recently, many different kinds of iodinated contrast media have been used. However, it is not clear whether differences exist in AEs among various LOCM (Park et al., 2018). Also, it is controversial whether iso-osmolar media are safer than LOCM on the topic of risk for contrast-induced nephropathy. Previous studies have evaluated various LOCM versus an iso-osmolar media iodixanol, but have shown conflicting results (Motosugi et al., 2016). Therefore, it is important to assess whether the risks associated with these media are class-specific, osmolality based, or individual medium-specific. Different iodinated contrast media may have different biological characteristics. In fact, knowledge of different safety profiles of individual iodinated contrast media is essential (Wu et al., 2016).

Therefore, the objectives of this paper is to determine identify characteristics of iodinated contrast media, types of iodinated contrast media, the associated adverse events, and the management of such associated adverse events.

\subsection{Statement of the Problem}

The processes of diagnosis and intervention based on contrast media are performed with increasing frequency. The patient population subjected to these procedures is progressively older and has more comorbid conditions (Park et al., 2018; Morzycki et al., 2017). Therefore, contrast media continue to cause concern among patients, referring clinicians, and radiologists because of their widespread use and the rare but potentially important adverse events (Tung, 2006).

Intravenous iodinated contrast media are commonly used with CT to evaluate disease and to determine treatment response (Morzycki et al., 2017). Although patients have benefited from their use, iodinated contrast media historically have been denied or delayed in patients with reduced kidney function due to the perceived risks of Contrast-Induced Acute Kidney Injury (CI-AKI) (Wu et al., 2016). This is important because denying patients diagnostic testing that is indicated in a timely fashion creates potential for indirect harm related to delayed diagnosis and misdiagnosis.

However, clinical decision making in patients potentially at risk is often fraught with confusion, uncertainty, and heterogeneity (Motosugi et al., 2016; Park et al., 2018; Barr et al., 2016; Rusandu et al., 2020). This is due in part to shifting perceptions regarding the true risks of modern contrast media, improvements in scientific methodology used to study these adverse events, incomplete penetrance of new knowledge into scientific practice, latent bias related to historical precedent, uncertainty regarding the interpretation of recently conducted well-controlled observational studies, and differences in recommendations across radiology and medical subspecialties (Imai et al., 2018).

It is significant to recognize that in clinical practice, a multitude of factors are used to determine whether intravenous contrast media should be administered (e.g., probability and necessity of an accurate diagnosis, alternative methods of diagnosis, risks of misdiagnosis, expectations about kidney functional recovery, allergic-like reaction risk) (Davenport et al., 2020). Decisions are rarely based on a single consideration (e.g., risk of an adverse event specifically related to kidney impairment). Consequently, these statements should be considered in the context of the entire clinical scenario. 


\subsection{Research Questions}

This paper aims to answer the following questions:

- What are the uses of iodinated contrast media?

- What are the clinical pharmacology properties of iodinated contrast media?

- What are the adverse effects of iodinated contrast media?

- How can the adverse effects of iodinated contrast media be managed?

\subsection{Methodology}

This paper uses the descriptive approach that involves a review of the literature published on iodinated contrast media in terms of their uses, clinical pharmacology, adverse effects, and their management. The review is based on the studies and researches published on iodinated contrast media with the aim to elaborate on this concept and elicit its distinct features.

\section{Literature Review}

\subsection{Iodinated Contrast Media}

Iodinated contrast agents have been in use since the 1950s to facilitate radiographic imaging modalities. Physicians in almost all specialties will either administer these agents or care for patients who have received these drugs (Jascinth et al., 2016; Park et al., 2018). Different iodinated contrast agents vary greatly in their properties, uses, and toxic effects. Therefore, clinicians should be at least superficially familiar with the clinical pharmacology, administration, risks, and adverse effects associated with iodinated contrast agents (Pasternak \& Williamson, 2012).

Contrast agents have long been used for the imaging of anatomic boundaries and to explore normal and abnormal physiologic findings. These agents have included colorimetric contrast agents (e.g., methylene blue and indocyanine green) and fluorescent contrast agents (e.g., fluorescein) (Morzycki et al., 2017). However, the introduction of increasingly faster and more discriminating radiographic imaging techniques has resulted in the need for radiationattenuating contrast agents that can be used in traditional radiographic imaging or, more recently, in subtraction imaging, both of which can be projected and rotated in three dimensions (Motosugi et al., 2016).

By far the most successful and widely applied contrast agents in use today are the Iodinated Contrast Agents (ICAs), first introduced into clinical practice in the 1950s. It is estimated that approximately 75 million doses of ICAs are given worldwide each year (Christiansen, 2005). The ICAs fall into 4 broad groups, each possessing unique chemical, physical, and biologic properties. These various ICAs are needed to address the demands of a wide variety of imaging modalities as shown in table 1 (Cohan et al, 2013).

Given the frequent use of ICAs and the diversity of images and patients requiring an ICA, it is not surprising that physicians from virtually every specialty will encounter patients scheduled to receive, or who have recently received, an ICA (Park et al., 2018). Because the ICAs collectively have the potential to meaningfully alter patient physiologic features, produce immunologic reactions, and affect patient outcomes (in concert with, or independent of, other disease processes), it is important that all physicians have an appreciation for the indications for, selection of, and consequences of ICA use. For these reasons, we provide a primer on ICA use for the non-radiologist. 
Table 1 Indications for Use of Iodinated Contrast Media

\begin{tabular}{|c|c|}
\hline Intravascular & Intra-arterial \\
\hline Intravenous & Angiocardiography \\
\hline Computed tomography & Computed tomography \\
\hline Digital subtraction angiography & Coronary angiography \\
\hline Intravenous urography & Pulmonary angiography \\
\hline Venography (phlebography) & Aortography \\
\hline Inferior vena cava and its tributaries & Visceral and peripheral arteriography \\
\hline Superior vena cava and its tributaries & Digital subtraction angiography \\
\hline Extremities & Central nervous system \\
\hline Other venous sites & Cerebral, vertebral, and spinal angiography \\
\hline \multicolumn{2}{|l|}{ Epidural venography } \\
\hline \multicolumn{2}{|l|}{ Intrathecal } \\
\hline \multicolumn{2}{|c|}{ Myelography (myelographic nonionic only) } \\
\hline \multicolumn{2}{|c|}{ Cisternography (myelographic nonionic only) } \\
\hline \multicolumn{2}{|l|}{ Other } \\
\hline \multicolumn{2}{|c|}{ Oral, rectal, or ostomy - gastrointestinal tract } \\
\hline \multicolumn{2}{|l|}{ Conventional fluoroscopy } \\
\hline \multicolumn{2}{|l|}{ Computed tomography } \\
\hline \multicolumn{2}{|l|}{ Therapeutic uses } \\
\hline \multicolumn{2}{|l|}{ Body cavity use } \\
\hline \multicolumn{2}{|l|}{ Herniorrhaphy } \\
\hline \multicolumn{2}{|l|}{ Peritoneography } \\
\hline \multicolumn{2}{|l|}{ Vaginography } \\
\hline \multicolumn{2}{|l|}{ Hysterosalpingography } \\
\hline \multicolumn{2}{|l|}{ Arthrography } \\
\hline \multicolumn{2}{|c|}{ Endoscopic retrograde cholangiopancreatography } \\
\hline \multicolumn{2}{|c|}{ Cholangiography } \\
\hline \multicolumn{2}{|l|}{ Nephrostography } \\
\hline \multicolumn{2}{|l|}{ Pyelography - antegrade, retrograde } \\
\hline \multicolumn{2}{|l|}{ Urethrography - voiding, retrograde } \\
\hline \multicolumn{2}{|l|}{ Cystography } \\
\hline \multicolumn{2}{|l|}{ Sialography } \\
\hline \multicolumn{2}{|l|}{ Ductography (breast) } \\
\hline \multicolumn{2}{|l|}{ Miscellaneous } \\
\hline \multicolumn{2}{|l|}{ Sinus tract injection } \\
\hline Cavity delineation & \\
\hline
\end{tabular}




\subsection{Pharmacology of Iodinated Contrast Media}

All ICAs share a similar function group-a tri-iodinated benzene ring (Figure 1). Iodine plays a key role in the attenuation of x-rays. The atomic radius of a covalently bonded iodine atom is approximately 133 picometers, which falls within the range of the wavelengths of x-rays: 10 to 10,000 picometers; thus, x-rays are easily attenuated by the iodine atoms. Furthermore, 3 iodine atoms covalently bonded to a benzene ring offer 2 major advantages: (1) 3 large atoms located in such close proximity increase the effective molecular size, thus attenuating longer-wavelength $\mathrm{x}$-rays, and (2) covalent bonding to a stable organic functional group (i.e., benzene) reduces the risk of toxic effects from free iodide.

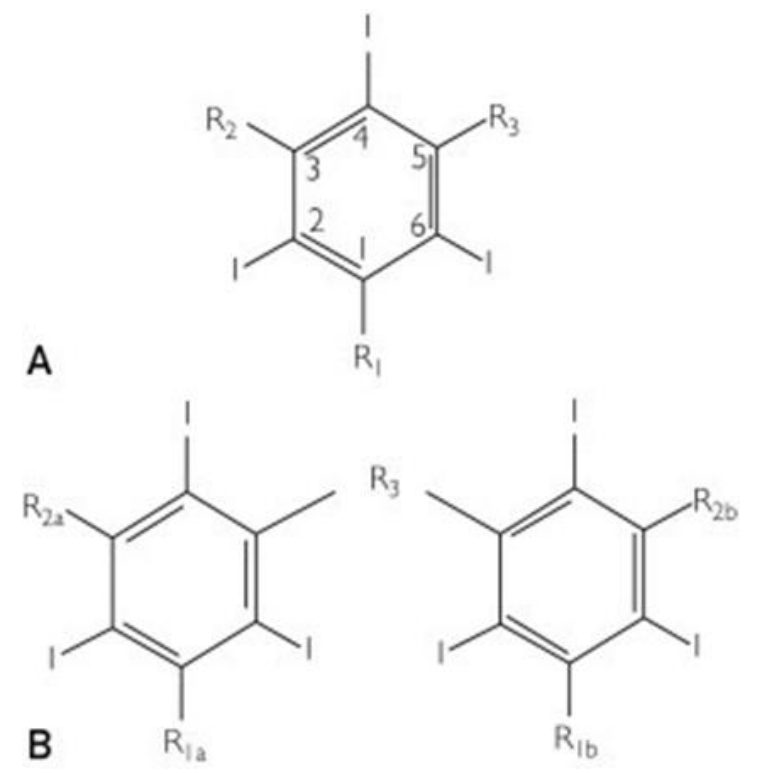

Figure 1 Basic Molecular Structural Units of Iodinated Contrast Agents. Source: (Pasternak \& Williamson, 2012).

Two major chemical variations result in 4 classes of ICAs (Figure 2). Compounds consist of either 1 tri-iodinated benzene ring (i.e., monomers) or 2 tri-iodinated benzene rings linked by an organic functional group (i.e., dimers). In addition, ionic tendency is governed by the presence (i.e., ionic) or absence (i.e., nonionic) of a carboxylate (-C00-) functional group contained on an organic side chain. Typically, because the carboxylate moiety adds a net negative charge to the molecule, these anionic agents are usually available as salts of sodium, calcium, or methylglucamine cations. Hence, the four major classes of iodinated contrast agents are as follows:

- Ionic monomer: single tri-iodinated benzene ring with a carboxylate-containing benzene substituent.

- Ionic dimer: two linked tri-iodinated benzene rings in which at least 1 carboxylate-containing group is substituted on at least 1 benzene ring.

- Nonionic monomer: single tri-iodinated benzene ring without a carboxylate-containing benzene substituent.

- Nonionic dimer: two linked tri-iodinated benzene rings that do not contain a carboxylate functional group within any benzene substituent. 


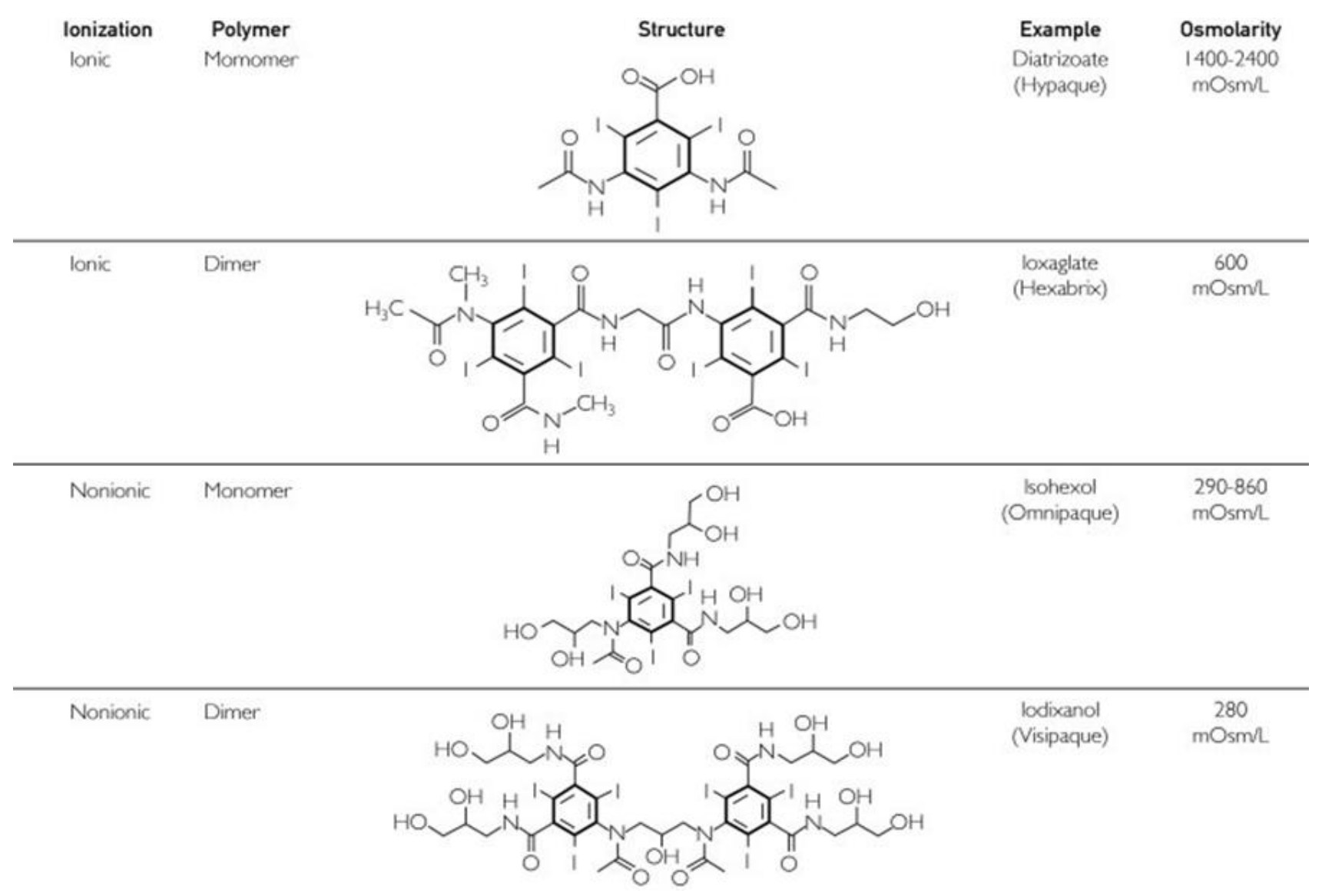

Figure 2 Properties of the 4 Classes of iodinated Contrast Agents. Source: (Pasternak \& Williamson, 2012).

The ICAs among the 4 groups have differing properties, clinical uses, and toxicity profiles, and to some degree these differences influence which types of agents are used by the imaging department for specific indications (Yamaguchi et al., 2017). For example, unlike nonionic agents, which are uncharged, the charged ionic species tend to disrupt the electrical potential of cell membranes, accounting for their increased toxicity (Thomsen \& Morcos, 2000). Also, ionic monomers have the weakest ability to attenuate $\mathrm{x}$-rays and thus need to be administered in high concentrations that are hyperosmolar (approximate osmolarity $=1500-2000 \mathrm{mOsm} / \mathrm{L}$ ) compared with blood (approximate osmolarity = 280-290 mOsm/L) (Han et al., 2019). As such, ionic monomers are also referred to as high-osmolarity agents. Lowosmolarity agents include ionic dimers and nonionic monomers with osmolarities in the range of 290 to $860 \mathrm{mOsm} / \mathrm{L}$. Nonionic dimers are iso-osmolar with blood, with an osmolarity of $290 \mathrm{mOsm} / \mathrm{L}$ (Kurihara et al., 2015).

All ICAs demonstrate low protein binding. Distribution from the intravascular compartment to highly perfused organs, such as brain, liver, and kidney, is rapid, whereas distribution to less perfused organs and tissues, such as bone and fat, is much slower (Imai et al., 2018). As such, the half-life of redistribution of the intravascular compartment is still rapid (2-5 minutes for most agents). Currently, no available agent undergoes clinically significant metabolism because all are eliminated unchanged by the kidneys via glomerular filtration with no significant tubular reabsorption (Katzberg, 1997).The elimination half-life of most agents falls within the range of 90 to 120 minutes in patients with normal renal function and can be delayed, on the order of weeks, in patients with renal insufficiency (Barr et al., 2016).

\subsection{Clinical Uses of ICM}

Because the administration of these media carries some increased risk for patients in certain clinical settings, it is important to weigh risks and benefits of contrast administration and choose the appropriate type and amount of contrast for each patient. Ideally, patients should be informed of these risks. In patients at high risk for an adverse event, the need for administration of an ICA should be considered, as well as alternative imaging modalities or the use of other contrast agents, such as gadolinium (Rusandu et al., 2020; Dona et al., 2020). Although the various ICAs differ in a number of physical and chemical properties, such as viscosity, osmolarity, and immunogenicity, their imaging characteristics are entirely based on the ability to attenuate x-rays (Lim et al., 2020). The ability of an ICA to attenuate $\mathrm{x}$-rays depends on the number of iodine molecules present in the tissue to be imaged and is inversely related to the $\mathrm{x}$ - 
ray energy used for imaging. Therefore, the type and amount of contrast media used for a given purpose also depend on the imaging characteristics needed. In general, there are three routes of administration for ICAs: intravascular, enteric, and direct injection (Brockow, 2020; Han et al., 2019):

\subsubsection{Intravascular Contrast Administration}

Intravascular administration of contrast is by far the most common use of iodinated contrast media and can be further subdivided into intra-arterial and intravenous injection. Intra-arterial injection is the primary method of contrast delivery used in diagnostic catheter angiography and catheter-directed arterial intervention, such as percutaneous angioplasty and stent placement. Factors that affect the choice of type and amount of contrast to be administered include viscosity, iodine concentration, and osmolarity (Yamaguhi et al., 2017; Kurihara et al., 2015).

The imaging modality used for most intra-arterial injections is fluoroscopy. This modality requires higher rates of contrast administration to opacify the target vessels (up to $30 \mathrm{~mL} / \mathrm{s}$ ) as opposed to intravenous injections used for computed tomography (CT) scanning (typically 2-6 mL/s) (Imai et al., 2018). Therefore, viscosity plays a significant role in the delivery of intra-arterial contrast media for angiography, and contrast agents are routinely warmed to $37^{\circ} \mathrm{C}$ so that adequate flow rates can be attained during catheter injection. In addition, iodine concentration can be an important factor for adequate opacification, and the use of high-iodine contrast media can be helpful, particularly in larger patients. Finally, osmolarity of intra-arterial contrast media has been shown to affect patient comfort during extremity angiography (Barr et al., 2016). For this reason, iso-osmolar contrast is frequently used for upper- and lower-extremity runoff studies.

Intravenous contrast administration for the purpose of CT scanning is the most common use of iodinated contrast media. Intravenous injection of contrast is also used for studies of the genitourinary tract, such as intravenous pyelography, and the venous system, such as direct venography; however, these studies have been decreasing in use during the last two decades, whereas the number of contrast-enhanced CT scans has increased significantly during that same period (Dona et al., 2020).

According to Lim et al (2020), the complications of intravascular injection of iodinated contrast include anaphylactoid contrast reaction and contrast-induced nephropathy. An additional risk of intravenous contrast injection is contrast media extravasation. Although uncommon, contrast extravasation can lead to local edema and erythema. Rarely, serious local effects, such as skin and subcutaneous ulceration and tissue necrosis, can occur.

\subsubsection{Enteric Contrast Administration}

Although oral or enteric contrast examinations traditionally are performed with barium suspensions, iodinated contrast media is also frequently used for this purpose (Brockow, 2020). In general, iodinated contrast is preferred to barium when there is a risk of bowel leak or obstruction because extraintestinal barium can cause inflammation and adhesions. Diatrizoate meglumine (GastroView) and diatrizoate sodium (Hypaque) are examples of iodinated contrast used for fluoroscopic examinations, typically at a $20 \%$ concentration. These agents can also be used in a more dilute form for CT examinations (usually $2 \%$ to $3 \%$ concentration) (Rusandu et al., 2020). Similarly, nonionic contrast agents, such as iohexol (Omnipaque), can be used for oral administration. At the dilutions used for CT scanning, these agents are nearly tasteless when dissolved in water (Imai et al., 2018).

\subsubsection{Direct Contrast Injection}

Direct injection of iodinated contrast comes in two basic forms: injection via percutaneous needle access, such as direct arthrography, and injection via an indwelling catheter or tube, such as cystography or sonography (Yamaguchi et al., 2017). Contrast injection of this type differs from intravascular injection in that the contrast is not rapidly cleared by the kidneys after image acquisition but is evacuated back through the catheter or by natural drainage. In some cases, such as articular injection and myelography, the contrast is absorbed slowly back into the body via the lymphatic system (Han et al., 2019).

As with enteric contrast administration, contrast reactions have been reported but are extremely rare. Contrast-related complications from such procedures are far more frequently associated with adverse local reactions (Barr et al., 2016). Therefore, the contrast preparation for each of these procedures should be specifically formulated for the appropriate indication. 


\subsection{Adverse Effects of Iodinated Contrast Media and their Management}

\subsubsection{Risks Associated with Iodinated Contrast Media}

Iodinated contrast media (CM) are routinely used to enhance imaging of neoplastic lesions. They are crucial for accurate depiction of the tumor, monitoring response to treatment, and assessing possible recurrence of malignant lesions (Morzycki et al., 2016). Unfortunately, there are potential risks associated with the administration of CM, and adverse reactions may occur. In addition, CM may interact with some of the drugs and clinical tests used in the management of patients with oncological disease (Motosugi et al., 2016).

Reactions to CM can be divided into general and renal adverse effects. General reactions are subdivided into acute (those that develop within one hour of CM administration) and delayed (those that develop after one hour but less than a week after CM administration) (Wu et al., 2016). Acute reactions can be divided into minor, intermediate, and severe lifethreatening reactions. The minor reactions include flushing, nausea, arm pain, pruritus, vomiting, headache, and mild urticarial (Jascinth et al., 2016). Patients suffering from reduced renal function or conditions causing reduction of renal perfusion may develop contrast media nephrotoxicity (CMN) (Morcos, 2008). Consideration should be given to alternative imaging techniques that do not require injection of iodinated CM (Park et al., 2018).

The use of gadolinium-based CM has been suggested for radiographic examinations as an alternative to iodinated CM in patients with renal impairment (Morzycki et al., 2017). Contrast media may interfere with the pharmacokinetics of other drugs, particularly those that are eliminated from the body through the kidneys. Iodinated contrast media can cause reduction of renal function, particularly in patients with preexisting reduced renal function (Motosugi et al., 2016).

\section{Adverse Effects of ICM}

Adverse effects from the administration of iodinated intravenous contrast media vary from mild reactions to rare but severe life-threatening situations (Wu et al., 2016).

\subsubsection{Contrast-Induced Acute Kidney Injury (Ci-AKI)}

Contrast-Induced Acute Kidney Injury (CI-AKI) has replaced other terminology such as contrast nephrotoxicity, Contrastinduced Nephropathy (CIN) or Radiocontrast Nephropathy (RCN). CI-AKI is the third leading cause of hospitalacquired acute renal failure (Kagan \& Sheikh-Hamad, 2010). It is an iatrogenic disease, which may cause long term morbidity or death. CI-AKI is defined when one of the following criteria is met:

- $\quad$ serum creatinine rises by $\geq 26 \mu \mathrm{mol} / \mathrm{l}$ within 48 hours after administration of contrast medium compared to baseline creatinine values;

- $\quad$ serum creatinine rises $\geq 1.5$ fold from the baseline value, which is known or presumed to have occurred within one week after administration of iodinated contrast medium;

- urine output is $<0.5 \mathrm{ml} / \mathrm{kg} /$ hour for more than 6 consecutive hours;

- when alternative explanations for renal impairment have been excluded.

These variable definitions make it difficult to predict the long-term clinical outcome of CI-AKI. CI-AKI is both an adverse effect that may permanently impair renal function, increase the length of hospital stay and hospital costs as well as a predictor of future adverse cardiovascular events and mortality (RCR, 2015).

\subsubsection{Acute Reactions}

Acute reactions are those which take place within 60 minutes of the administration of ICM. The majority of acute reactions are anaphylactoid, are not dose-dependent, and involve the release of histamine along with other active biological chemicals. Acute reactions may be classified as mild, moderate and severe (RCR, 2015):

- $\quad$ Mild Reactions: may be experienced by up to three percent of patients following iodinated contrast media administration. Such reactions usually resolve without the need for any treatment, and include nausea, pruritus, vomiting, marked urticaria, headache, and mild urticaria (RCR, 2015; ACR, 2013; RANZCR, 2009; Bettmann, 2004). 
- Moderate Reactions: may require specific treatment, and include severe vomiting, marked urticaria, bronchospasm, facial/laryngeal oedema and vasovagal attacks (RCR, 2015; ACR, 2013; RANZCR, 2009; Bettmann, 2004).

- Severe Reactions: occur in $0.04 \%$ to $0.004 \%$ of iodinate contrast media administrations, with the risk of death being rare. Severe reactions include hypovolaemic shock, respiratory arrest, cardiac arrest, pulmonary oedema and convulsions (RCR, 2015; ACR, 2013; RANZCR, 2009; Bettmann, 2004).

\subsubsection{Delayed Reactions}

Delayed reactions, which account for less than four percent of reactions, take place between one hour to one week after the administration of iodinated contrast media. Delayed reactions most commonly present as skin reactions with a maculopapular rash. Less frequent skin reactions include angioedema, urticaria and erythema (RCR, 2015; ACR, 2013; RANZCR 2009; Bettmann, 2004).

\subsection{Managing the Risks of Iodinated Contrast Media}

True CI-AKI is defined when there is a permanent reduction in renal function as opposed to a temporary alteration of renal function post iodinated contrast medium administration (Kurihara et al., 2015). True CI-AKI is rare and usually confined to individuals with pre-existing renal dysfunction. The risk of $\mathrm{CI}-\mathrm{AKI}$ is $0.6 \%$ to $2.6 \%$ in the general population, but rises to $4.7 \%$ in patients with pre-existing renal impairment (Imai et al., 2018). Renal impairment, measured best by Estimated Glomerular Filtration Rate (eGFR), is the only risk factor predictive of CI-AKI. Patients who have a high risk for CI-AKI need to be identified prior to iodinated contrast media administration through measurements of egFR. Renal impairment is considered in patients with an egFR $<60 \mathrm{ml} / \mathrm{mim} / 1.73 \mathrm{~m} 2$ (Dona et al., 2020).

However, the Contrast Media Safety Committee of the European Society of Urogenital Radiology, in their updated guidelines, agreed that the risk of CI-AKI is lower after intravenous than after intra-arterial administration of contrast media (Rusandu et al., 2020). They considered that only patients with an eGFR of $<45 \mathrm{ml} / \mathrm{min} / 1.73 \mathrm{~m} 2$ are at risk from CI-AKI after intravenous administration. Other risk factors, such as age over 75 years, diabetes, metformin use, congestive heart failure, gout and collagen vascular disease should also be considered (Richenberg, 2012; Stacul, 2011).

To reduce the risk of CI-AKI, eGFR measurements should be available for all nonemergency cases. An eGFR within the previous three months is satisfactory for patients in a stable condition. Patients with known renal disease should have an eGFR measurement from the previous seven days, which can be compared against a baseline. In the presence of chronic kidney disease (eGFR $<40 \mathrm{ml} / \mathrm{min} / 1.73 \mathrm{~m} 2$ ), one should consider temporarily stopping Angiotensin-Converting Enzyme (ACE) inhibitors and angiotensin II receptor blockers. Consultation with a nephrologist has been shown to be beneficial for patients at risk of CI-AKI (RCR, 2015; Lewington et al, 2020).

To date, the best known preventive measure to reduce the risk of CI-AKI is hydration. The smallest dose of low osmolar non-ionic monomeric or isoosmolar non-ionic dimeric contrast medium should be used. There is insufficient evidence to suggest routine use of any other pharmacological agent in attempting to reduce CI-AKI (RCR 2015).

Mild reactions to iodinated contrast are relatively common, but are mostly self-limited and of no consequence. Severe reactions, although rare, can occur in the absence of any specific risk factor with any type of contrast media (RCR, 2015; ACR, 2013; RANZCR, 2009). Therefore the aims during the administration of iodinated contrast are to:

- Ensure that the administration of iodinated contrast is appropriate for the patient and the clinical indication;

- $\quad$ Reduce the possibility of a reaction;

- Be fully prepared to promptly recognize and treat a reaction if this occurs.

General safety considerations during the administration of iodinated contrast include the availability of an appropriately trained doctor, who can promptly deal with a severe reaction. Those administering iodinated contrast media should be able to identify the symptoms of severe reactions. Patients should be well hydrated prior to iodinated contrast media administration. Facilities for the treatment of acute reactions should be readily available and regularly checked. No patient should be left unsupervised within the first five minutes post administration of iodinated contrast. It is good practice for patients to remain on the premises for at least 15 minutes post administration of iodinated contrast as, based on evidence, most severe reactions occur during this period (RCR, 2015). This period should be 30 minutes for individuals who are at an increased risk of adverse reactions. All types of reactions should be reported and documented for future reference (RCR, 2015; ACR 2013; Richenberg, 2012; Owen et al. 2014; RANZCR 2009; Bettmann 2004). 
Whilst there is always a potential risk for all individuals undertaking iodinated contrast media examinations, there should be a system whereby individuals who pose an increased risk can be identified so that adequate planning and precautionary measures can be taken before their administration. In the presence of an increased risk, the decision about iodinated contrast media administration should be taken by the supervising radiologist based on adequate information (Royal College of Radiologists, 2015; American College of Radiology, 2013; Owen et al., 2014; RANZCR 2009). For all such cases, if administration of iodinated contrast is still considered necessary, the following general precautionary measures should be taken:

- Supervise patient continuously;

- Leave cannula in place for at least 30 minutes post administration;

- $\quad$ Ensure availability of emergency drugs and equipment.

If iodinated contrast administration is necessary during pregnancy, there is a small risk of thyroid suppression to the fetus, and therefore a thyroid function test should be performed during the first week after birth (Royal College of Radiologists, 2015).

A small percentage of iodinated contrast is passed on to breast milk in lactating mothers. However, no specific precaution is necessary and mothers may continue to breastfeed their infants without any significant risk (Royal College of Radiologists, 2015).

Preparation for the treatment of iodinated contrast media reactions must include preparation for the whole variety of possible reactions and include availability of appropriately trained personnel, medications and equipment (RANZCR, 2009)

In terms of the administration of iodinated contrast media, quality assurance relates to the systematic monitoring and evaluation of the various aspects of administration, to ensure that required standards of quality are being met and continuously maintained. To ensure patient safety during the administration of iodinated contrast, resuscitation equipment, and medications for the treatment of complications should be made readily available and regularly checked and maintained (RANZCR, 2009).

All personnel who perform venepuncture for the administration of iodinated contrast should be well trained in: (Royal College of Radiologists, 2015)

- Venepuncture procedures and have received formal certification of their competence;

- The recognition of iodinated contrast reactions and the procedures for their treatment;

- Cardiopulmonary resuscitation (CPR).

The competencies of personnel should be updated and maintained through training programs as part of their Continuous Professional Development (CPD).

\section{Conclusion}

Recognition of the early signs of iodinated contrast media adverse effects, the risk of reactions to pre-existing conditions, taking adequate precautions to minimize risks and provision of prompt and adequate treatment ensures optimal patient safety and care. Adverse reactions to ICAs are common; however, severe reactions are rare. Most severe acute reactions and contrast-induced nephropathy occur with high-osmolarity ionic monomeric ICAs. Delayed reactions are most common after nonionic dimers. The best means to treat all adverse reactions is to prevent them from occurring. Identification of patients who are at risk for adverse reactions and minimizing risk by following preventive measures or using alternative (i.e., ICA independent) diagnostic techniques can be helpful in reducing the incidence of adverse effects. Furthermore, physicians responsible for ordering imaging tests that require the use of ICAs should consider addressing risks, benefits, and alternatives with their patients given the potential for adverse outcomes associated with this class of drugs. Equipment and drugs used to treat severe and life-threatening reactions should be readily available in all clinical locations where ICAs are administered. 


\section{Compliance with ethical standards}

\section{Disclosure of conflict of interest}

All authors declare that no conflict of interest is exist.

\section{References}

[1] American College of Radiology (2013). ACR manual on contrast media. Version 9. Reston, VA: American College of Radiology.

[2] Barr ML, Chiu HK, Li N, Yeh MW, Rhee CM, Casillas J, Iskander PJ, Leung AM. Thyroid Dysfunction in Children Exposed to Iodinated Contrast Media. The Journal of clinical endocrinology and metabolism. 2016; 101(6): 23662370.

[3] Bettmann MA. Frequently asked questions: iodinated contrast agents. Radiographics. 2004; 24(suppl_1): S3S10.

[4] Brockow K. Reduced iodinated contrast media dose and injection speed for CT: how much does this decrease the risk of a hypersensitivity reactions? Quantitative imaging in medicine and surgery. 2020; 10(2): 537-540.

[5] Christiansen C. X-ray contrast media: an overview. Toxicology. 2005; 209(2): 185-187.

[6] Cohan RH, Dillman JR, Hartman RP, Jafri SZ, Wang CK, Newhouse JH. American College of Radiology Manual on Contrast Media. American College of Radiology. Version. 2013; 9.

[7] Davenport MS, Perazella MA, Yee J, Dillman JR, Fine D, McDonald RJ, Rodby RA, Wang CL, Weinreb JC. Use of Intravenous Iodinated Contrast Media in Patients with Kidney Disease: Consensus Statements from the American College of Radiology and the National Kidney Foundation. Kidney Medicine. 2020; 2(1): 85-93.

[8] Dona I, Bogas G, Salas M, Testera A, Moreno E, Laguna JJ, Torres MJ. Hypersensitivity Reactions to Multiple Iodinated Contrast Media. Frontiers in Pharmacology. 2020; 11: 575437.

[9] Han S, Yoon SH, Lee W, Choi YH, Kang DY, Kang HR. Management of Adverse Reactions to Iodinated Contrast Media for Computed Tomography in Korean Referral Hospitals: A Survey Investigation. Korean journal of radiology. 2019; 20(1): 148-157.

[10] Imai K, Ikeda M, Satoh Y, Fujii K, Kawaura C, Nishimoto T, Mori M. Contrast enhancement efficacy of iodinated contrast media: Effect of molecular structure on contrast enhancement. European journal of radiology open. 2018; 5: 183-188.

[11] Jascinth ASL, Reddy CU, Mouli GSC, Sudharshan R, Khan AF, Anitha M. Contrast Agents In computed tomography: A Review. Journal of Applied Dental and Medical Sciences. 2016; 2: 2.

[12] Kagan A, Sheikh-Hamad D. Contrast-induced kidney injury: focus on modifiable risk factors and prophylactic strategies. Clinical cardiology. 2010; 33(2): 62-66.

[13] Katzberg RW. Urography into the 21st century: new contrast media, renal handling, imaging characteristics, and nephrotoxicity. Radiology. 1997; 204(2): 297-312.

[14] Kurihara O, Takano M, Uchiyama S, Fukuizumi I, Shimura T, Matsushita M, Komiyama H, Inami T, Murakami D, Munakata R, Ohba T, Hata N, Seino Y, Shimizu W. Microvascular resistance in response to iodinated contrast media in normal and functionally impaired kidneys. Clinical and experimental pharmacology \& physiology. 2015; 42(12): 1245-1250.

[15] Lewington A, MacTier R, Hoefield R, Sutton A, Smith D, Downes M. Prevention of contrast induced acute kidney injury (CI-AKI) in adult patients. KIDNEYS. 2020; 9(1): 58-60.

[16] Lim E, Jang JH, Yoon D, Min YG, Kim HH. Does Exposure to Computed Tomography Contrast Media Increase Risk of End-Stage Renal Disease? Medical Science Monitor: International Medical Journal of Experimental and Clinical Research. 2020; 26: e921303.

[17] Morcos SK. Adverse reactions to iodinated contrast media. In Cancer imaging. 2008; 97-106.

[18] Morzycki A, Bhatia A, Murphy KJ. Adverse reactions to contrast material: a Canadian update. Canadian Association of Radiologists Journal. 2017; 68(2): 187-193. 
[19] Motosugi U, Ichikawa T, Sano K, Onishi H. Acute adverse reactions to nonionic iodinated contrast media for CT: prospective randomized evaluation of the effects of dehydration, oral rehydration, and patient risk factors. American Journal of Roentgenology. 2016; 207(5): 931-938.

[20] Owen RJ, Hiremath S, Myers A, Fraser-Hill M, Barrett BJ. Canadian Association of Radiologists consensus guidelines for the prevention of contrast-induced nephropathy: update 2012. Canadian Association of Radiologists Journal. 2014; 65(2): 96-105.

[21] Park SJ, Kang DY, Sohn KH, Yoon SH, Lee W, Cho YH, Kang HR. Immediate mild reactions to CT with iodinated contrast media: strategy of contrast media readministration without corticosteroids. Radiology. 2018; 288(3): 710-716.

[22] Pasternak JJ, Williamson EE. Clinical pharmacology, uses, and adverse reactions of iodinated contrast agents: a primer for the non-radiologist. Mayo Clinic proceedings. 2012; 87(4): 390-402.

[23] Richenberg J. How to reduce nephropathy following contrast-enhanced CT: a lesson in policy implementation. Clinical radiology. 2012; 67(12): 1136-1145.

[24] Royal Australian and New Zealand College of Radiologists (RANZCR). RANZCR Guidelines for Iodinated Contrast Administration.2009

[25] Royal College of Radiologists. Standards for intravascular contrast agent administration to adult patients. Royal College of Radiologists..2010

[26] Rusandu A, Sjøvold BH, Hofstad E, Reidunsdatter RJ. Iodinated contrast media and their effect on thyroid function - Routines and practices among diagnostic imaging departments in Norway. Journal of medical radiation sciences. 2020; 67(2): 111-118.

[27] Stacul F, van der Molen AJ, Reimer P, Webb JA, Thomsen HS, Morcos SK, Heinz-Peer G. Contrast induced nephropathy: updated ESUR contrast media safety committee guidelines. European radiology. 2011; 21(12): 2527-2541.

[28] Thomsen HS, Morcos SK. Radiographic contrast media. BJU international. 2000; 86(s 1): 1-10.

[29] Tung HK. Risks of iodinated contrast media—what you should know. Medical Bulletin. 2006; 11(6): 15-17.

[30] Wu YW, Leow KS, Zhu Y, Tan CH. Prevention and management of adverse reactions induced by iodinated contrast media. Ann Acad Med Singapore. 2016; 45(4): 157-164.

[31] Yamaguchi N, Fukushima Y, Yamaguchi A, Nagasawa N, Taketomi-Takahashi A, Suto T, Tsushima Y. Sensation of smell and taste during intravenous injection of iodinated contrast media in CT examinations. The British journal of radiology. 2017; 90(1069): 20160629. 